\title{
Dietary vitamin intake and risk of metabolic syndrome among centenarians in China
}

\author{
ZHIQING PENG ${ }^{1}$, YANYAN WANG ${ }^{2}$, XIANYONG HUANG ${ }^{2}$, QIAO ZHU ${ }^{3}$, \\ YALI ZHAO $^{3}$, HENGGE XIE ${ }^{4}$ and JIANJUN WU ${ }^{2}$ \\ Departments of ${ }^{1}$ Clinical Nutrition, ${ }^{2}$ Healthcare and ${ }^{3}$ Central Laboratory, \\ Hainan Hospital of Chinese PLA General Hospital, Sanya, Hainan 572000; ${ }^{4}$ Department of Geriatric Neurology, \\ The Second Medical Center, Chinese PLA General Hospital/National Clinical Research Center for Geriatric Diseases, \\ Beijing 100853, P.R. China
}

Received April 3, 2020; Accepted July 17, 2020

DOI: $10.3892 /$ etm.2020.9537

\begin{abstract}
The present study aimed to investigate dietary vitamin intake levels and their association with the prevalence of obesity, hypertension, dyslipidemia and hyperglycemia in centenarians in China. From June 2014 to December 2016, a total of 992 centenarians aged $>99$ years (177 males and 815 females; age range, 100-115 years) were enrolled through household visits in the cities and rural areas of Hainan province. Details regarding food intake were recorded by continuous collection of 7-day food frequency and 24-h dietary review, and dietary vitamin intake levels were calculated according to the Chinese Food Composition Table. The deficiency rates of vitamin $\mathrm{A}(\mathrm{VA}), \mathrm{VE}, \mathrm{VB}_{1}, \mathrm{VB}_{2}$, niacin and $\mathrm{VC}$ among the centenarians were relatively high and the prevalence of metabolic syndrome (MS) was 53.67\% (519/967). The dietary intake levels of VA, VE and PP were significantly higher among the healthy centenarians than among the centenarians with MS $(\mathrm{P}<0.05)$. Compared with the lowest quartiles $\left(\mathrm{Q}_{1}\right)$ of dietary vitamin intake, higher dietary intake levels of VA $\left(\mathrm{Q}_{4}\right)$ [odds ratio $(\mathrm{OR})=0.72 ; 95 \% \mathrm{CI}: 0.38,0.99], \mathrm{VE}\left(\mathrm{Q}_{3}\right)(\mathrm{OR}=0.61$; $95 \% \mathrm{CI}=0.36,0.88)$ and $\mathrm{VB}_{2}\left(\mathrm{Q}_{4}\right)(\mathrm{OR}=0.51 ; 95 \% \mathrm{CI}: 0.32,0.81)$ were associated with a reduced risk of hypertension $(\mathrm{P}<0.05)$. However, higher dietary intake levels of VA, VE, VB $\mathrm{VB}_{2}$ and PP were associated with increased risks of central obesity, hyperglycemia and low high-density lipoprotein cholesterol levels. A high prevalence of MS and vitamin deficiency were detected
\end{abstract}

Correspondence to: Mr. Jianjun Wu, Department of Healthcare, Hainan Hospital of Chinese PLA General Hospital, 20 Jianglin Road, Linwang, Haitang Bay, Sanya, Hainan 572000, P.R. China E-mail: wujj301@163.com

Abbreviations: CHCCS, China Hainan Centenarian Cohort Study; VA, vitamin A; PP, niacin; FBG, fasting blood glucose; TG, triglyceride; HDL-C, high-density lipoprotein cholesterol; LDL-C, low-density lipoprotein cholesterol; MS, metabolic syndrome

Key words: China Hainan Centenarian Cohort Study, dietary vitamin, centenarians, metabolic syndrome, China among the centenarians and these two items were associated with each other. It was indicated that specific vitamins are necessary for certain centenarians.

\section{Introduction}

With the global aging phenomenon becoming a current social trend, there is an increasing focus on the study of dietary vitamin intake of centenarians. Vitamins are trace organic compounds required for maintaining the normal functioning of the human body. They have a vital role in promoting the synthesis and degradation of major nutrients in metabolic processes. Dietary vitamins cannot be synthesized and stored in excess in the human body and a small amount of vitamins provided by consumed food is able to meet the requirements of the body (1). Extensive research has demonstrated that vitamins have a certain preventive effect on various chronic diseases (2), but there is a lack of studies focusing on the relationship between dietary vitamin intake and longevity, particularly among centenarians.

Among centenarians, due to the degeneration of teeth, the type and amount of total dietary intake is largely limited and decreased $(3,4)$. Furthermore, degeneration of the digestion and absorption system leads to reduced absorption of vitamins. Thus, it is reasonable to doubt that the dietary vitamin intake is a primary influence among centenarians. However, only limited previous evidence focused on dietary vitamins and metabolic syndrome. Most of the previous studies focused on the individual vitamin levels and dietary vitamins, including vitamin $\mathrm{D}$ (VD) and $\mathrm{VB}_{12}$, and metabolic disorders (5-7). VD deficiency has been frequently reported among centenarians in terms of health status and VD supplementation may be necessary for aged individuals, which would be helpful to slow down the processes of 'fragilization' occurring in those subjects (8). A growing body of recent evidence suggested that the levels of several vitamins, including $\mathrm{VD}$ and $\mathrm{VB}_{12}$, are significantly associated with metabolism in the human body (9). In addition, several studies evaluated metabolic syndrome (MS) in the offspring of centenarians and indicated that offspring of long-lived parents have a lower prevalence of MS than their partners despite having a comparable body composition $(10,11)$. 
However, evidence on the various dietary vitamins and metabolism in centenarians is still lacking. Therefore, the present study aimed to evaluate the dietary vitamin intake of centenarians in Hainan Province and to explore the association of dietary vitamin intake levels with the prevalence of obesity, hypertension, dyslipidemia and hyperglycemia based on data obtained from the China Hainan Centenarian Cohort Study (CHCCS), a prospective cohort study. Furthermore, it was attempted to collect evidence for developing dietary nutritional interventions, preventing and controlling chronic diseases and improving the quality of life of centenarians.

\section{Materials and methods}

Participants. The current study analyzed data from CHCCS, one of the largest centenarian health interdisciplinary prospective cohort studies conducted in China from June 2014 to December 2016. Details of the CHCCS have been described previously (3). This study was conducted in Hainan province, an area with the highest density of centenarians in China and an iodine-sufficient region with abundant seafood provision (12). A total of 1,002 centenarians were recruited in the study. Among them, 874 centenarians (150 males and 724 females) without any missing data were included in the final analysis. The present study was approved by the Ethics Committee of the Hainan branch of the Chinese People's Liberation Army General Hospital (Sanya, China; no. 301hn11201601). Each participant signed an informed consent form after receiving information about the contents of the research. The present cross-sectional survey was performed among the centenarians from all cities, rural areas, farms and islands (including Sansha City) of Hainan province in 2014.

Measurements. Physical examination was performed and demographic information was collected by a professional nurse or trained investigator in a face-to-face interview. Demographic information included age, sex, ethnicity (Han, Li and other), education level (illiterate, primary school and middle school or higher), marital status (married, widowed, divorced or never married), family history of chronic diseases (cancer, cardiovascular diseases, hypertension, diabetes, stroke, mental diseases and dementia), smoking status (never smoker, former smoker and current smoker), alcohol consumption status (current, former and never), physical activity (yes or no) and tea consumption (frequent, occasionally and never). A dietary survey was also conducted to collect information on nutritional risk screening and dietary habits. Details about daily food intake (staple and non-staple foods, snacks and drinks) were recorded by the participants or their family members for 7 consecutive days through a 24-h dietary review. The proportion of vegetable and fruit consumption was also included and recorded in their daily food intake questionnaire. Based on the $24 \mathrm{~h}$ dietary review, dietary intake levels of VA, $\mathrm{VE}, \mathrm{VB} 1$, niacin (PP) and VC were calculated according to the Chinese Food Composition Table (13). Data on body height, body weight, calf circumference, hip circumference, waist circumference (WC) and systolic/diastolic blood pressure were obtained by measurement during on-site medical examination.

Venous blood samples were collected from each participant in the seated position after at least $5 \mathrm{~min}$ rest by an experienced nurse, transported in cold storage to the clinical laboratory and assayed within $4 \mathrm{~h}$ of collection. Laboratory blood tests were performed to determine fasting blood glucose (FBG), serum triglyceride (TG), high-density lipoprotein cholesterol (HDL-C) and low-density lipoprotein (LDL-C) levels; these were measured using enzymatic assays (Roche Products, Ltd.) on a fully automatic biochemical autoanalyzer (COBAS c702; Roche Products, Ltd.). All assays were performed by qualified technicians who were blinded to the clinical data.

Diagnostic criteria. The diagnostic criteria for MS were set according to the National Cholesterol Education Program Adult Treatment Group III (14). In brief, adults with $\geq 3$ conditions among the following were diagnosed with MS: i) Abdominal obesity: $\mathrm{WC} \geq 85 \mathrm{~cm}$ in males or $\mathrm{WC} \geq 80 \mathrm{~cm}$ in females (15); ii) hypertension: Blood pressure $\geq 140 / 90 \mathrm{mmHg}$ and confirmed diagnosis of hypertension or receiving treatment (16); iii) TG: $\geq 1.70 \mathrm{mmol} / \mathrm{l}$; iv) low HDL-C: $<1.04 \mathrm{mmol} / 1$ (40 mg/l) in males or $<1.30 \mathrm{mmol} / 1(50 \mathrm{mg} / \mathrm{dl})$ in females; and v) high FBG: $\geq 6.1 \mathrm{mmol} / 1$.

Statistical analysis. Dietary vitamin intake levels are expressed as the median (interquartile range) for data with a non-normal distribution, and the Mann-Whitney U test was performed for comparing participants with and without MS (MS and non-MS groups, respectively). After dividing the participants into four categories according to the quartiles of dietary vitamin intake levels of the non-MS group $\left(\mathrm{Q}_{1}-\mathrm{Q}_{4}\right.$; the group with the lowest quartile was used as the reference group), logistic regression models were used to explore the association between MS and its components and dietary vitamin intake. The odds ratio (OR) of MS and 95\% CI were calculated using logistic regression models after adjusting for sex, marital status, physical activity, smoking status, alcohol consumption, family history of chronic diseases and daily total energy intake. All data analyses were performed using the Statistical Package for Social Sciences (version 20.0; IBM, Corp.). $\mathrm{P}<0.05$ was considered to indicate statistical significance.

\section{Results}

General characteristics. A total of 992 centenarians [177 males (17.84\%) and 815 females (82.16\%); age range, $100-115$ years] completed the medical examinations and dietary surveys and 967 of them underwent laboratory blood tests (17). Among them, 519 centenarians were diagnosed with MS [prevalence: $53.67 \%$ (519/967)]. Compared with the recommended intake or appropriate intake levels according to the Chinese Dietary Reference Intake (DRI, 2013 edition) (18), the dietary deficiency rates of $\mathrm{VB}_{2}, \mathrm{VB}_{1}, \mathrm{PP}, \mathrm{VA}, \mathrm{VC}$ and $\mathrm{VE}$ among these centenarians were $87.55,80.13,67.63,45.81,30.26$ and $23.59 \%$, respectively (data not shown).

Comparison of vitamin intake between the MS and non-MS groups. The dietary intake levels of VA, VE and PP in the non-MS group were significantly higher than those in the MS group, with differences in mean vitamin intake as follows: $61.83 \mu \mathrm{g}$ retinol activity equivalents/day, $5.56 \mathrm{mg} \alpha$-tocopherol equivalents/day and $1.88 \mathrm{mg}$ niacin equivalents/day, respectively $(\mathrm{P}<0.05$ for all; Table I). Although the dietary intake 
Table I. Dietary intake levels of vitamins among centenarians in Hainan province.

\begin{tabular}{|c|c|c|c|c|}
\hline Vitamin & $\mathrm{RNI} / \mathrm{AI}$ & MS (n=519) & Non-MS (n=448) & P-value \\
\hline $\mathrm{VA}(\mu \mathrm{g} \mathrm{RAE} / \mathrm{d})$ & 700 & $444.11(275.02,582.26)$ & $505.94(474.43,616.14)$ & 0.027 \\
\hline $\mathrm{VE}(\mathrm{mg} \alpha-\mathrm{TE} / \mathrm{d})$ & 14 & $12.18(9.00,16.85)$ & $17.74(10.45,20.67)$ & 0.001 \\
\hline $\mathrm{VB}_{1}(\mathrm{mg} / \mathrm{d})$ & 1.2 & $0.65(0.45,0.90)$ & $0.87(0.48,9.09)$ & 0.192 \\
\hline $\mathrm{VB}_{2}(\mathrm{mg} / \mathrm{d})$ & 1.2 & $0.49(0.22,0.98)$ & $0.59(0.39,1.91)$ & 0.239 \\
\hline $\mathrm{PP}$ (mg NE/d) & 10 & $7.43(5.40,11.25)$ & $9.31(5.10,15.92)$ & 0.026 \\
\hline $\mathrm{VC}(\mathrm{mg} / \mathrm{d})$ & 100 & $79.71(53.09,98.70)$ & $88.50(55.98,121.90)$ & 0.674 \\
\hline
\end{tabular}

$\mathrm{VA}, \mathrm{VB}_{1}, \mathrm{VB}_{2}$ and PP refer to the RNI for females aged $>80$ years according to dietary reference intake. Values are expressed as the median (interquartile range). P-values were calculated by using Mann-Whitney U tests. RNI, recommended nutrient intake; AI, appropriate intake; MS, metabolic syndrome; VA, vitamin A; pp, niacin; d, day; RAE, retinol activity equivalents; TE, $\alpha$-tocopherol equivalents; NE, niacin equivalents.

levels of $\mathrm{VB}_{1}, \mathrm{VB}_{2}$ and $\mathrm{VC}$ were higher in the non-MS group than in the MS group, the difference was not significant (P>0.05 for all).

Association between MS and its components and dietary vitamin intake. Logistic regression models were used to evaluate the associations between the quartiles of vitamin intake and MS or its components after adjusting for sex, marital status, sexual activity status, physical activity, smoking status, alcohol consumption, family history of chronic diseases and daily total energy intake (Table II). It was indicated that higher dietary intake levels of VA $\left(Q_{4}\right)$ were significantly associated with a reduced risk of hypertension $(\mathrm{OR}=0.72$, 95\% CI: $0.38,0.99)$, and the linear association between VA intake and hypertension was significant $(\mathrm{P}<0.05)$. Although higher dietary intake levels of $\mathrm{VA}\left(\mathrm{Q}_{3}\right)$ among the centenarians were associated with a higher risk of abdominal obesity $(\mathrm{OR}=1.08,95 \% \mathrm{CI}: 1.00,2.40)$ and hyperglycemia $(\mathrm{OR}=1.45$, $95 \%$ CI: $1.00,1.83)$, the linear trends were not significant ( $\mathrm{P}>0.05$ for both).

Higher dietary intake levels of VE $\left(\mathrm{Q}_{3}\right)$ were associated with a decreased risk of hypertension $(\mathrm{OR}=0.61,95 \% \mathrm{CI}: 0.36$, $0.88)$ and the linear trend of their association was significant $(\mathrm{P}<0.01)$ among the centenarians. Although higher dietary intake levels of $\mathrm{VE}$ were also associated with a higher risk of hyperglycemia $(\mathrm{OR}=1.51,95 \% \mathrm{CI}$ : $0.27,2.20)$, the P-value was $>0.05$. A significant linear decrease in the risk of MS with high dietary intake levels of VE was also observed, although the OR for $\mathrm{Q}_{2}-\mathrm{Q}_{4}$ was not significant.

Higher dietary intake levels of $\mathrm{VB}_{2}\left(\mathrm{Q}_{4}\right)$ were associated with a reduced risk of hypertension $(\mathrm{OR}=0.51,95 \% \mathrm{CI}: 0.32$, $0.81)$ and an increased risk of low HDL-C levels ( $\mathrm{OR}=1.40$, 95\% CI: 1.04, 2.23), and the linear trends for $\mathrm{VB}_{2}$-hypertension and $\mathrm{VB}_{2}$-low HDL-C were significant $(\mathrm{P}<0.05$ for both). Elevated dietary intake levels of $\mathrm{PP}\left(\mathrm{Q}_{3}\right)$ were associated with an increased risk of abdominal obesity $(\mathrm{OR}=1.85,95 \% \mathrm{CI}$ : $1.20,3.13)$, but the association was not significant $(\mathrm{P}>0.05)$.

Although dietary intake levels of $\mathrm{VE}, \mathrm{VB}_{1}, \mathrm{VB}_{2}$ and $\mathrm{PP}$ exhibited a significant linear association with the risk of MS, compared with the lowest quartile, we did not found any significant association of dietary $\mathrm{VE}, \mathrm{VB}_{1}, \mathrm{VB}_{2}$ and $\mathrm{PP}$ with MS. The dietary intake levels of VA demonstrated significant associations with the odds of waistline, hypertension, and hyperglycemia, only the VA-hypertension association was with a significant linear trend.

\section{Discussion}

Using data from CHSS, a large centenarian cohort study performed in China, the present study discovered that high dietary intake levels of VA, VE, $\mathrm{VB}_{2}$ and $\mathrm{PP}$ were associated with high risks of central obesity, hyperglycemia and low HDL-C levels, whereas high dietary intake levels of VA and VE were associated with a reduced risk of hypertension.

The present results indicated relatively high deficiency rates of $\mathrm{VB}_{1}, \mathrm{VB}_{2}$ and $\mathrm{VC}$ among the centenarians in Hainan province, whereas the dietary intake levels of VA, VE and PP were sufficient. These results are consistent with those of a study on dietary vitamin intake among the elderly in the Zhejiang and Nanjing provinces $(19,20)$. In comparison, the dietary intake level of VA was relatively high among the centenarians in Hainan province. This was possibly due to the specific diet of Hainan island, located in southernmost of China. The food is comparatively lighter, less oily and more mildly seasoned than that in mainland China. Seafood and various tropical fruits and vegetables, which contain high vitamin levels, predominate the menu. A previous systematic review reported that the dietary intake of VE was significantly insufficient in the worldwide elderly population (21), whereas the majority of the elderly in Hainan province met or exceeded the VE intake levels recommended by the Chinese Nutrition Society (22), which may be due to the sufficient intake of VE-rich seafood in Hainan province. In addition, regarding the highly insufficient vitamin intake rates, the elderly are prone to insufficient dietary vitamin intake due to the decrement of energy intake, digestion, absorption, synthesis and metabolic decomposition secondary to the natural decline of organ function, as well as due to chronic diseases, nutritional deficiencies and poor eating habits.

In the present study, the non-MS group had higher dietary intake levels of $\mathrm{VA}, \mathrm{VE}, \mathrm{VB}_{1}, \mathrm{VB}_{2}, \mathrm{PP}$ and $\mathrm{VC}$ than the MS group. After adjusting for potential confounders, including sex, ethnicity, marital status and daily total energy intake, it was revealed that the dietary intake levels of VA, VE and PP exerted a protective effect on MS. This conclusion is consistent with that of a Polish study on Alzheimer's disease, 
Table II. Logistic regression analysis of MS and its components among centenarians in Hainan province.

A, VA

\begin{tabular}{|c|c|c|c|c|c|}
\hline Vitamins & $\mathrm{Q}_{1}$ & $\mathrm{Q}_{2}$ & $\mathrm{Q}_{3}$ & $\mathrm{Q}_{4}$ & P-value \\
\hline MS & 1 & $1.21(0.70,1.79)$ & $1.09(0.66,1.72)$ & $1.15(0.67,1.85)$ & 0.576 \\
\hline Waistline & 1 & $1.27(0.87,1.88)$ & $1.08(1.00,2.40)$ & $1.25(0.73,2.28)$ & 0.136 \\
\hline Hypertension & 1 & $0.86(0.50,1.17)$ & $0.65(0.36,1.05)$ & $0.72(0.38,0.99)$ & 0.011 \\
\hline Hyperglycemia & 1 & $1.12(0.72,1.75)$ & $1.45(1.00,1.83)$ & $1.16(0.73,1.85)$ & 0.505 \\
\hline Hypertriglyceridemia & 1 & $0.97(0.62,1.53)$ & $1.29(0.83,2.01)$ & $1.10(0.69,1.76)$ & 0.471 \\
\hline Low HDL-C & 1 & $1.72(0.97,3.04)$ & $1.15(0.62,2.10)$ & $1.41(0.75,2.64)$ & 0.429 \\
\hline
\end{tabular}

$\mathrm{B}, \mathrm{VE}$

\begin{tabular}{|c|c|c|c|c|c|}
\hline Vitamins & $\mathrm{Q}_{1}$ & $\mathrm{Q}_{2}$ & $\mathrm{Q}_{3}$ & $\mathrm{Q}_{4}$ & P-value \\
\hline MS & 1 & $1.16(0.73,1.86)$ & $1.11(0.67,1.83)$ & $1.05(0.59,1.88)$ & 0.025 \\
\hline Waistline & 1 & $1.30(0.83,2.06)$ & $1.30(0.81,2.09)$ & $1.36(0.79,2.32)$ & 0.823 \\
\hline Hypertension & 1 & $0.79(0.52,1.22)$ & $0.61(0.36,0.88)$ & $0.63(0.38,1.04)$ & 0.002 \\
\hline Hyperglycemia & 1 & $1.31(0.84,2.04)$ & $1.47(0.80,2.01)$ & $1.51(0.27,2.20)$ & 0.141 \\
\hline Hypertriglyceridemia & 1 & $0.96(0.62,1.50)$ & $1.12(0.70,1.77)$ & $0.96(0.57,1.62)$ & 0.670 \\
\hline Low HDL-C & 1 & $1.28(0.71,2.33)$ & $1.25(0.67,2.33)$ & $1.16(0.58,2.30)$ & 0.829 \\
\hline
\end{tabular}

$\mathrm{C}, \mathrm{VB}_{1}$

\begin{tabular}{|c|c|c|c|c|c|}
\hline Vitamins & $\mathrm{Q}_{1}$ & $\mathrm{Q}_{2}$ & $\mathrm{Q}_{3}$ & $\mathrm{Q}_{4}$ & P-value \\
\hline MS & 1 & $1.28(0.79,2.02)$ & $0.81(0.47,1.37)$ & $0.91(0.53,1.84)$ & 0.017 \\
\hline Waistline & 1 & $1.30(0.82,2.06)$ & $1.53(0.93,2.52)$ & $1.60(0.89,2.87)$ & 0.734 \\
\hline Hypertension & 1 & $0.83(0.54,1.28)$ & $0.87(0.54,1.39)$ & $0.68(0.39,1.17)$ & 0.101 \\
\hline Hyperglycemia & 1 & $1.09(0.70,1.69)$ & $0.94(0.58,1.52)$ & $0.93(0.53,1.63)$ & 0.222 \\
\hline Hypertriglyceridemia & 1 & $1.11(0.71,1.72)$ & $1.03(0.64,1.67)$ & $0.99(0.56,1.74)$ & 0.215 \\
\hline Low HDL-C & 1 & $1.19(0.66,2.15)$ & $1.77(0.40,1.50)$ & $1.23(0.58,2.60)$ & 0.931 \\
\hline
\end{tabular}

$\mathrm{D}, \mathrm{VB}_{2}$

\begin{tabular}{|c|c|c|c|c|c|}
\hline Vitamins & $\mathrm{Q}_{1}$ & $\mathrm{Q}_{2}$ & $\mathrm{Q}_{3}$ & $\mathrm{Q}_{4}$ & $\mathrm{P}$-value \\
\hline MS & 1 & $0.89(0.56,1.42)$ & $0.67(0.41,1.10)$ & $1.00(0.57,1.76)$ & 0.011 \\
\hline Waistline & 1 & $1.18(0.74,1.87)$ & $1.45(0.91,2.32)$ & $1.64(0.96,2.79)$ & 0.490 \\
\hline Hypertension & 1 & $1.03(0.67,1.59)$ & $0.71(0.46,1.11)$ & $0.51(0.32,0.81)$ & 0.021 \\
\hline Hyperglycemia & 1 & $0.81(0.52,1.26)$ & $0.73(0.46,1.14)$ & $0.81(0.48,1.35)$ & 0.122 \\
\hline Hypertriglyceridemia & 1 & $0.92(0.59,1.43)$ & $0.75(0.47,1.19)$ & $1.20(0.72,2.00)$ & 0.953 \\
\hline Low HDL-C & 1 & $1.05(0.60,1.84)$ & $0.76(0.41,1.42)$ & $1.40(1.04,2.23)$ & 0.017 \\
\hline
\end{tabular}

$\mathrm{E}, \mathrm{PP}$

\begin{tabular}{|c|c|c|c|c|c|}
\hline Vitamins & $\mathrm{Q}_{1}$ & $\mathrm{Q}_{2}$ & $\mathrm{Q}_{3}$ & $\mathrm{Q}_{4}$ & P-value \\
\hline MS & 1 & $1.53(0.94,2.47)$ & $1.26(0.74,2.15)$ & $1.73(0.92,3.27)$ & 0.015 \\
\hline Waistline & 1 & $1.42(0.89,2.27)$ & $1.85(1.20,3.13)$ & $1.79(0.89,3.42)$ & 0.852 \\
\hline Hypertension & 1 & $1.19(0.77,1.85)$ & $0.91(0.57,1.46)$ & $0.80(0.46,1.41)$ & 0.084 \\
\hline Hyperglycemia & 1 & $1.11(0.71,1.74)$ & $0.98(0.60,1.59)$ & $0.98(0.55,1.75)$ & 0.032 \\
\hline Hypertriglyceridemia & 1 & $1.30(0.80,1.45)$ & $0.79(0.59,1.71)$ & $1.22(0.57,1.89)$ & 0.529 \\
\hline Low HDL-C & 1 & $1.12(0.57,1.70)$ & $0.64(0.48,1.48)$ & $1.70(0.63,3.31)$ & 0.016 \\
\hline
\end{tabular}

According to dietary vitamin intake, the participants were divided into 4 groups as quartile arrays $\mathrm{Q}_{1}, \mathrm{Q}_{2}, \mathrm{Q}_{3}$ and $\mathrm{Q}_{4} ; \mathrm{Q}_{2}, \mathrm{Q}_{3}$ and $\mathrm{Q}_{4}$ were based on $\mathrm{Q}_{1}$, and $\mathrm{P}$-values were calculated by logistic linear trend of MS and its components $\mathrm{Q}_{1}-\mathrm{Q}_{4}$. Values are expressed as the odds ratios (95\% CI). MS, metabolic syndrome; VA, vitamin A; PP, niacin; HDL-C, high-density lipoprotein cholesterol. 
which concluded that VA and VE possibly have a protective role against chronic diseases and aging $(23,24)$. Furthermore, the present large-sample study on the risk of cardiovascular disease demonstrated that VA, VE and $\mathrm{VB}_{2}$ exerted protective effects against hypertension, whereas $\mathrm{VB}_{2}$ was associated with an increased odds of low HDL-C levels. In addition, the current study demonstrated that VA and PP increased the risk of abdominal obesity.

According to the present results, dietary factors may be the primary causes of insufficient vitamin intake. There are several aspects that may potentially affect insufficient vitamin intake, such as the following: i) Due to the poor conditions of oral hygiene and residual teeth among the elderly, they frequently consume liquid and semiliquid foods, which directly leads to a lack of essential vitamin intake. ii) Due to their impaired chewing function, soft foods requiring a prolonged cooking time are preferred for centenarians; however, high-temperature processing destroys $\mathrm{VB}$ and $\mathrm{VC}$ present in the food. iii) Insufficient intake of vegetables and low frequency of fruit intake leads to multivitamin deficiency. iv) The majority of the elderly subjects experienced inconvenience in daily life due to their reduced mobility and perceived a lack of care by their family. The long-term consumption of a simple diet negatively affected the optimum dietary intake of multiple vitamins and nutrients. v) The local economic status of Hainan province suggests that it is an underdeveloped region with limited medical care facilities. During the past decade, the proportion of the centenarian population in Hainan province has increased by $250 \%(25,26)$. This suggests that the government should focus more on the quality of life of centenarians and provide the required social and medical support.

The CHCCS is a large and well-designed prospective cohort study on centenarians in Hainan province in China (3), which is helpful to evaluate dietary vitamin intake and MS. To the best of our knowledge, the present study was the first to evaluate the association between dietary vitamins and metabolic syndromes in centenarians. The functional degeneration of the organs causes a large decrement in the uptake of the nutrients among centenarians. The uptake levels of nutrients among centenarians are difficult to evaluate. However, dietary vitamin intake levels are positively associated with the vitamin levels within human body, and dietary vitamin levels could partially represent uptake levels. Therefore, the question of whether dietary vitamin intake influences metabolic disease is important for centenarians. However, there are several limitations to this study. First of all, the present study was conducted in Hainan province, which is an island in the South of China. The dietary types and lifestyles are unique, which may limit the extension of the conclusions to other populations. Furthermore, the information on diet was based on questionnaires completed using face-to-face interviews. Due to the short term memory loss exhibited by the centenarians, recall bias may influence results. In addition, the cross-sectional design and unavailable residual confounders may not allow for a causal conclusion. Furthermore, social and economic factors were not taken into account and this may have been a source of bias. In addition, the present study only focused on several vitamins, including $\mathrm{VA}, \mathrm{VE}, \mathrm{VB}_{1}, \mathrm{VB}_{2}, \mathrm{PP}$ and $\mathrm{VC}$, and other vitamins were not evaluated, which should be performed in a further study. Finally, compared with blood vitamin levels in the centenarians, the dietary information may be less precise to reflect the vitamin levels in the human body.

In summary, the dietary vitamin intake levels of centenarians in Hainan province were generally low. As the proportion of the centenarian population is increasing, there is a requirement to provide a specific DRI recommendation for them according to the general Chinese Dietary Guidelines. Although the dietary vitamin intake levels of centenarians were higher than other populations in other provinces, the intake levels were generally lower than normal adults. Thus, it may be recommended that centenarians should increase their intake of foods rich in VA, VE, and $\mathrm{VB}_{2}$. In addition, a reasonable intake of vitamin supplements is suggested for centenarians who are not able to achieve sufficient intake of vitamins only through their diet.

\section{Acknowledgements}

Not applicable.

\section{Funding}

The design of the study, collection, analysis and interpretation of data, and manuscript writing were funded by the Medical Technology Innovation Project of Sanya (grant no. 2017YW09), the National Natural Science Foundation of China (grant no. 81941021), National Key R\&D Program of China (grant no. 2018YFC2000400), National S\&T Resource Sharing Service Platform Project of China [grant no. YCZYPT(2018)07] and the General Hospital of PLA Medical Big Data R\&D Project (grant no. MBD2018030).

\section{Availability of data and materials}

The datasets used and/or analyzed during the present study are available from the corresponding author on reasonable request.

\section{Authors' contributions}

ZP designed the present study and drafted the manuscript. YW acquired and analyzed the data. XH designed the current study and performed a literature search. QZ and YZ acquired the data. HX acquired the samples. JW interpreted the data. HX and JW agreed to be accountable for all aspects of the work in ensuring that questions related to the accuracy or integrity of any part of the work are appropriately investigated and resolved. All authors read and approved the final manuscript.

\section{Ethics approval and consent to participate}

This study was approved by the Ethics Committee of the Hainan branch of the Chinese People's Liberation Army General Hospital (Sanya, China; no. 301hn11201601). Each participant signed an informed consent form after receiving information about the research contents.

\section{Patient consent for publication}

Not applicable. 


\section{Competing interests}

The authors declare that they have no competing interests.

\section{References}

1. Sun $\mathrm{CH}$ : Nutrients and foods Hygienics. People's Medical Publishing House Co. Ltd., Beijing, 2012.

2. Fletcher RH and Fairfield KM: Vitamins for chronic disease prevention in adults: Clinical applications. JAMA 287: 3127-3129, 2002.

3. He Y, Zhao Y, Yao Y, Yang S, Li J, Liu M, Chen X, Wang J, Zhu Q, Li X, et al: Cohort profile: The China Hainan Centenarian Cohort Study (CHCCS). Int J Epidemiol 47: 694-695, 2018.

4. He Y, Luan FX, Yao Y, Yang SS, Xie HG, Li J, Liu M, Wang JH, Wu L, Zhu Q, et al: China Hainan Centenarian Cohort Study: Study design and preliminary results. Zhonghua Liu Xing Bing Xue Za Zhi 38: 1292-1298, 2017 (In Chinese).

5. Vitezova A, Zillikens MC, van Herpt TT, Sijbrands EJ, Hofman A, Uitterlinden AG, Franco OH and Kiefte-de Jong JC: Vitamin D status and metabolic syndrome in the elderly: The Rotterdam Study. Eur J Endocrinol 172: 327-335, 2015.

6. Ford ES, Ajani UA, McGuire LC and Liu S: Concentrations of serum vitamin D and the metabolic syndrome among U.S. Adults. Diabetes Care 28: 1228-1230, 2005.

7. Thomas-Valdés S, Tostes MDGV, Anunciação PC, da Silva BP and Sant'Ana HMP: Association between vitamin deficiency and metabolic disorders related to obesity. Crit Rev Food Sci Nutr 57: 3332-3343, 2017

8. Hausman DB, Fischer JG and Johnson MA: Nutrition in centenarians. Maturitas 68: 203-209, 2011.

9. D'Ambrosio DN, Clugston RD and Blaner WS: Vitamin A metabolism: An update. Nutrients 3: 63-103, 2011.

10. Rozing MP, Westendorp RG, de Craen AJ, Frölich M, de Goeij MC, Heijmans BT, Beekman M, Wijsman CA, Mooijaart SP, Blauw GJ, et al: Favorable glucose tolerance and lower prevalence of metabolic syndrome in offspring without diabetes mellitus of nonagenarian siblings: The Leiden longevity study. J Am Geriatr Soc 58: 564-569, 2010.

11. Ostan R, Bucci L, Cevenini E, Palmas MG, Pini E, Scurti M, Vescovini R, Caruso C, Mari D, Vitale G, et al: Metabolic syndrome in the offspring of centenarians: Focus on prevalence, components, and adipokines. Age (Dordr) 35: 1995-2007, 2013.

12. Hao Z, Liu Y, Li Y, Song W, Yu J, Li H and Wang W: Association between Longevity and element levels in food and drinking water of typical Chinese Longevity Area. J Nutr Health Aging 20: 897-903, 2016.

13. Yang YX, Wang GY and Pan XC: China Food Composition. Peking University Medical Press, Beijing, 2002.
14. Grundy SM, Cleeman JI, Daniels SR, Donato KA, Eckel RH, Franklin BA, Gordon DJ, Krauss RM, Savage PJ, Smith SC Jr, et al: Diagnosis and management of the metabolic syndrome: An American Heart Association/National Heart, Lung, and blood institute scientific statement. Circulation 112: 2735-2752, 2005

15. Chen CM and Kong LZ: Guidelines for the Prevention and Control of Overweight and Obesity in Chinese Adults. People's Medical Publishing House Co. Ltd., Beijing, 2006.

16. Wang W: Guidelines for primary management of hypertension in China (2014 revision). Zhonghua Gao Xue Ya Za Zhi 23: 24-43, 2015 (In Chinese).

17. Han QX, Zhang D, Zhao YL, Liu L, Li J, Zhang F, Luan FX, Liu DW, Liu ZS, Cai GY, et al: Risk factors for hyperuricemia in Chinese Centenarians and Near-Centenarians. Clin Interv Aging 14: 2239-2247, 2019.

18. Society CN: Reference intake of dietary nutrients for Chinese residents. China Science Publishing \& Media Ltd., Beijing, 2014.

19. Zhou HT, Wang SK, Yin X, et al: Nutrition and health status of elderly population in Lishui district of Nanjing in China. Chong Qing Yi Xue Chongqing Med 43: 3333-3335, 2014.

20. Zhang RH, Zhou B, Zhu XH, Huang LC, Zhu YB and Ding GQ: The relationship between vitamin intake and metabolic syndrome in the elderly in Zhejiang Province in China. Acta Nutrimenta Sinica 38: 299-301, 2016.

21. Ter Borg S, Verlaan S, Hemsworth J, Mijnarends DM, Schols JM, Luiking YC and de Groot LC: Micronutrient intakes and potential inadequacies of community-dwelling older adults: A systematic review. Br J Nutr 113: 1195-1206, 2015.

22. Wang SS, Lay S, Yu HN and Shen SR: Dietary guidelines for chinese residents (2016): Comments and comparisons. J Zhejiang Univ Sci B 17: 649-656, 2016.

23. Raszewski G, Chwedorowicz R, Chwedorowicz A and Gustaw Rothenberg K: Homocysteine, antioxidant vitamins and lipids as biomarkers of neurodegeneration in Alzheimer's disease versus non-Alzheimer's dementia. Ann Agric Environ Med 23: 193-196, 2016.

24. Heart Outcomes Prevention Evaluation Study Investigators; Yusuf S, Dagenais G, Pogue J, Bosch J and Sleight P: Vitamin E supplementation and cardiovascular events in high-risk patients N Engl J Med 342: 154-160, 2000.

25. Population Census Office under the State Council of China, National Bureau of Statistics and Department of Population and Employment Statistics: Tabulation On the 2010 Population Census of the People's Republic of China by County. China Statistics Press, 2012.

26. Population Census Office under the State Council of China, National Bureau of Statistics and Department of Population S, Science and Technology Statistics: Tabulation On the 2010 Population Census of the People's Republic of China. China Statistics Press, 2002. 